\title{
Capillary haemangiomas: an approach to their management
}

\author{
M J Boyd, J R O Collin
}

\begin{abstract}
Twenty-five children with eyelid haemangiomas were reviewed. Fifteen patients with enlarging lesions thought to be at risk of causing amblyopia were treated with intralesional steroids as soon after presentation as possible. This appeared to reduce significantly the incidence of amblyopia. Surgery was reserved for older children in whom no further involution of the lesion was expected.
\end{abstract}

Capillary haemangiomas of the lid are a distinct clinical entity in children. They threaten vision by causing anisometropia, sensory deprivation, amblyopia, and strabismus.' Their management and its effectiveness have remained controversial owing to the high complication rate and uncertain success of any treatment. The tumours follow a characteristic clinical course, appearing shortly after birth, growing quickly in the first year of life, and then beginning a slow, involutional phase; $40 \%$ resolve by age 4 and $70 \%$ by age $7 .^{2}$ Despite this the risk of amblyopia has encouraged a variety of treatments to be used. Radiotherapy is associated with cataracts and cutaneous scarring, and sclerosing agents with scarring and unpredictability. Radon seeds and cryotherapy both cause cutaneous atrophy. Eventually intralesional steroids were tried, first as an addendum to a trial of systemic steroids by Zarem and Edgerton, ${ }^{3}$ then more extensively by Mazzola ${ }^{4}$ and Kushner. ${ }^{5}$

For 10 years we have been using intralesional steroids during the active phase of lesional growth and with adjunctive treatment (occlusion and glasses). Surgical debulking is done if necessary at a later inactive phase. We present our results on 25 patients over a 10 -year-period.

\section{Subjects and methods}

All the patients had capillary haemangiomas diagnosed by virtue of the history of onset and progression of disease and the appearance of the lesions. Seventeen of the 25 patients were female $(68 \%)$. Sixteen $(64 \%)$ were symptomatic within two weeks of birth and $23(92 \%)$ before three months. The patients presented to our clinic between the ages of 1 month and 7 years. Twenty of 25 patients' lesions ( $80 \%$ ) were on the upper lid. The largest and smallest axes of the lesions were measured, but computerised tomography was not routinely performed, and without this it was impossible to assess the variable bulk and volume of the lesions.

Treatment was thought to be warranted if the lesion threatened the visual axis or if there were signs suggesting amblyopia (strabismus or refractive error). In accordance with their severity some lesions were followed up expectantly; the children were refracted and treated with glasses and occlusion therapy as necessary. Other children, in whom amblyopia was a greater risk, received steroid injections. The routine injection consisted of $40 \mathrm{mg}$ of methylprednisolone and $4 \mathrm{mg}$ of dexamethasone sodium phosphate. The first was injected directly into the lesion, and the second, more soluble, was injected paralesionally. The direct intralesional injection was given deeply to prevent long-acting depotsteroid deposits from being visible under the skin. The paralesional injection was given subcutaneously round the periphery of the haemangioma. The injections were repeated three times at approximately sixweek intervals if there was some response but no gross decrease in the size of the lesion. Two patients had a total of four injections each.

Surgery consisted of lid debulking or adjusting lid height or contour and was done only after the tumour had passed into an inactive growth phase, usually after the age of six. Twenty two patients presented to us without any previous treatment, but three had initially undergone treatment elsewhere: one had a course of cryotherapy followed by one course of steroid injection; one had had two courses of cryotherapy; and one arrived with ptosis after surgical excision.

\section{Results}

Of the 25 patients 15 needed occlusion treatment, nine required glasses, and 15 had intralesional steroids. Of those not treated with steroids six had presented prior to its usage, two were children with lesions not warranting injections (too small or thought not to be at risk of causing amblyopia) and two presented after the active growth phase of the tumour had ended.

Twelve of the $15(80 \%)$ required only one or two injections, but one case needed three and another two needed four. The injections often initially caused some extravasation of blood. The patients were reviewed after four weeks when any haematoma had absorbed. A subjective assessment was made by asking the parents if they thought the lesion had regressed, and an objective assessment was made by measuring the major and minor axes of the lesion. The injections had a significant reducing effect in nine of $15(60 \%)$ cases (Figs 1A, B), questionable effect in four, and little or no effect in two (Figs 2). In those cases that responded there was a considerable reduction in subcutaneous vascularity as well as a reduction in bulk, but this was not easy to quantify. If subjectively and objectively there was a dramatic response, the injection was not repeated, but if there was only a partial improve- 


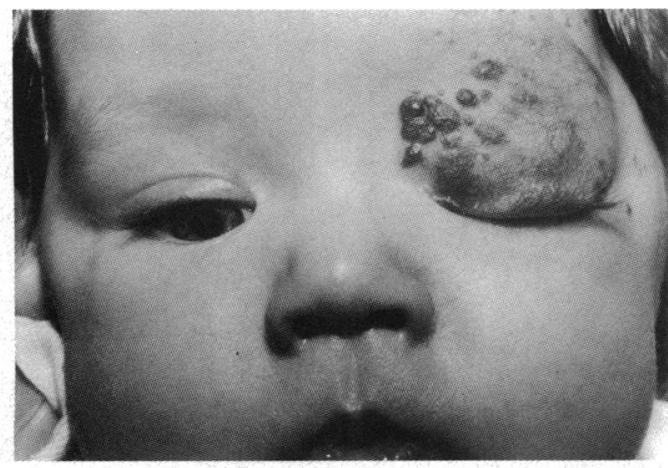

Figure 1A Four-month-old child with actively enlarging upper lid haemangioma.

ment the injection was repeated up to four times. If there was no response or the lesion actually increased in size despite the injection, it was not repeated. It was not possible to identify the factors which made it more likely that some lesions would respond while others would not. No systemic side effects from the steroids were noted. Follow-up for all patients was a mean of 1 year 11 months, being more than twice as long for the non-injected group - 1 year 4 months (range 3 months to 3 years) versus 2 years 10 months (range $1 \frac{1}{2}$ to 5 years).

The main reason for treating the patients, particularly with steroids, was the risk of amblyopia. Amblyopia is described as a unilateral or bilateral decrease in vision for which no obvious cause can be detected. ${ }^{6}$ It proved to be a major complication with these tumours, occurring in $64 \%$ of our cases $(16 / 25)$. It occurred in eight out of 10 cases $(80 \%)$ treated without steroids and in eight of $15(53 \%)$ receiving steroid injections. It was much more frequent with the upper lid lesions, being seen in 16 of 20 cases $(80 \%)$ compared with 1 of $5(20 \%)$ patients with lower lid lesions. This single case of lower lid haemangioma was associated with long-term dense amblyopia and was left with counting finger vision in the affected eye, but the lesion was very large (Fig 3). Although it was not possible to measure the lesions accurately, it was noted that, if the lesion was near the eyelid margin and the visual axis was threatened or occluded, amblyopia was common. We did not find that earlier injections gave better visual results than the later ones. The most amblyopic cases in the injected groups included both a child who had been injected at the earliest age ( 2 months) with an end vision of hand movements and the child who had been injected at the latest

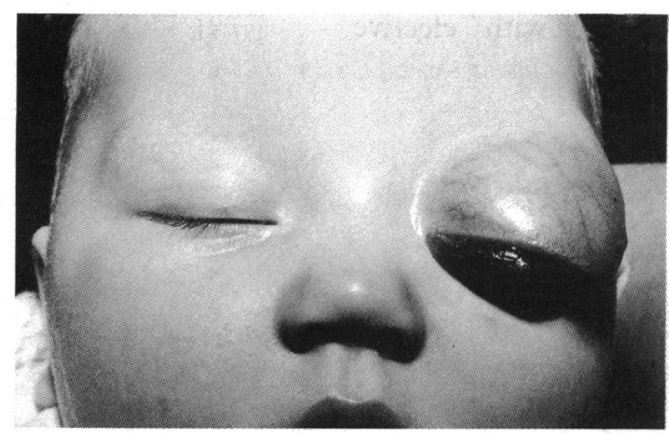

Figure 2A Three-month-old child with actively enlarging upper lid haemangioma.

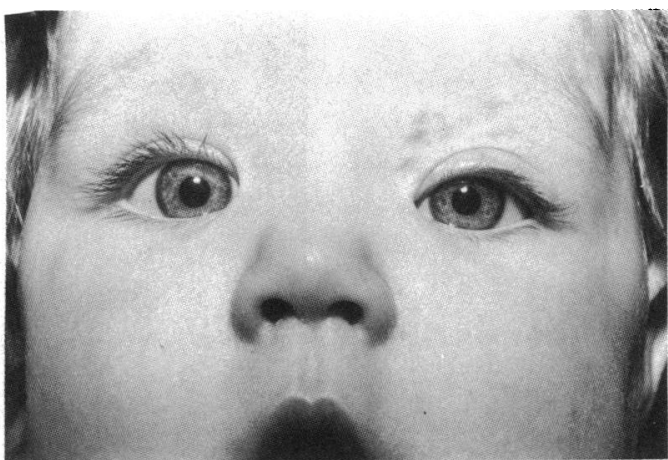

Figure IB Same child three months after steroid injection showing rapid resolution.

age $\left(2 \frac{1}{2}\right.$ years) with an end vision of counting fingers.

Six children had a total of eight operations at a mean age of $71 / 2$ years to correct the residual bulk of the lesion, entropion, ectropion, or ptosis. Five operations were debulking procedures via an anterior skin approach (with one including an anterior lid repositioning); one was a full-thickness lid resection (Fig 4), another was a ptosis repair (anterior levator resection), and the remaining operation was a skin crease reformation procedure. Postoperatively the results were satisfactory, though in two cases the skin crease was not exactly symmetrical and the ptosis was slightly overcorrected in one case and undercorrected in another.

\section{Discussion}

There have been several large studies reviewing either the complications or treatment of capillary haemangiomas of the lid. Stigmar et $\mathrm{al}^{7}$ reviewed 51 cases of eyelid haemangiomas and found 27 with ophthalmic complications: $44 \%$ developed amblyopia and $34 \%$ strabismus. They advocated a close follow-up with surgery, steroids, or $x$ rays if vision was threatened. Cosmetic repair was delayed until age 8 .

Haik et al' analysed their results of treating 101 eyelid haemangiomas. Clinical features included early recognition by the family ( $95 \%$ by 6 months of age) and female predominance $(61 \%)$ and superior lid involvement $(75 \%)$. Forty eight cases had long-term follow-up, with $60 \%$ showing amblyopia, usually secondary to sensory deprivation or anisometropia. Various types of treatment were used, but the type of treatment did not noticeably alter the cosmetic or functional outcome. Kushner ${ }^{8}$ was an early advocate

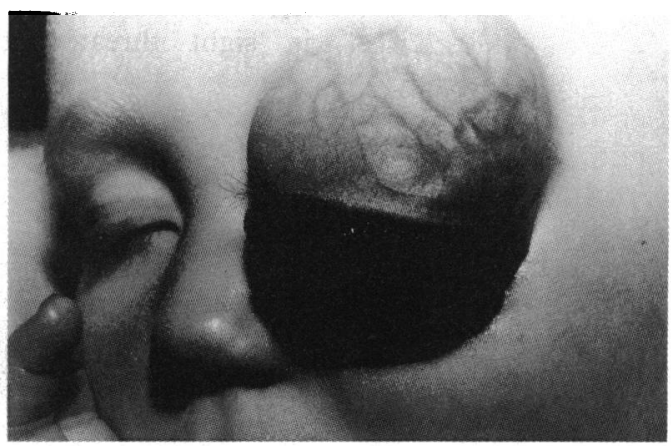

Figure 2B Same child one year after steroid injections showing progression of lesion despite treatment. 


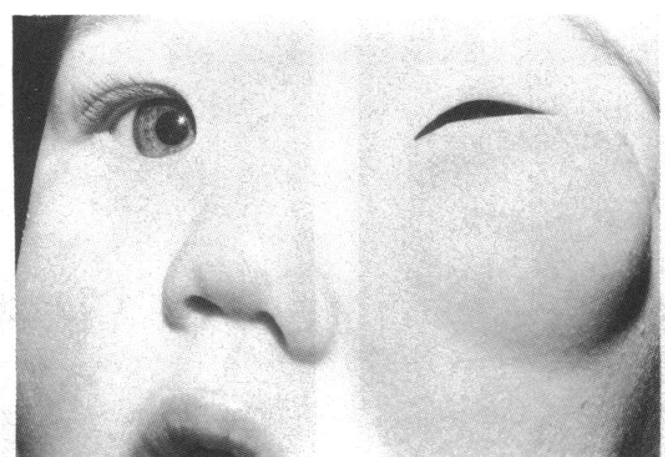

Figure 3 Two-year-old child with large lower lid haemangioma causing amblyopia.

of intralesional steroids and has reported his results of injecting 25 eyelid haemangiomas. Total resolution occurred in 16; seven showed less than $50 \%$ decrease in the size of the lesion, but enough resolution to prevent amblyopia. Only three cases failed to respond to treatment.

Our study confirms many of these findings. They include the high female predominance, predilection for the upper lid, and early clinical presentation. Amblyopia remains the foremost complication, occurring in $64 \%$ of the cases in our study. Its frequency emphasises the need for early treatment and early follow-up. There was a reduction in the frequency of amblyopia with the use of injectable steroids ( $80 \%$ down to $53 \%$ ), and this should become more pronounced as the follow-up becomes longer.

We elected to use the same dosage of intra- and perilesional steroids for all haemangiomas as described by Kushner. ${ }^{58}$ Bonavolonta et $a l^{9}$ adjusted the injectable steroid dose to the size of the haemangioma as determined by CT, but they could not prove that this method was more successful, and at least one case developed Cushing's syndrome. Our arbitrary approach with a common steroid dose irrespective of size or location of the tumour showed a successful method of managing this lesion without major complications.

Surgery in this study was limited to the inactive growth phase of the tumour. It was used in cosmetic debulking procedures and was routinely successful. It was not associated with excessing haemorrhage or rebound growth. Technically the skin crease and lid height were most difficult to predict postoperatively.

In conclusion, eyelid haemangioma remains a distinct clinical entity associated with a high risk of amblyopia and complications from treatment. A method of management has been described which includes an intralesional steroid injection in sight threatening lesions, with elective

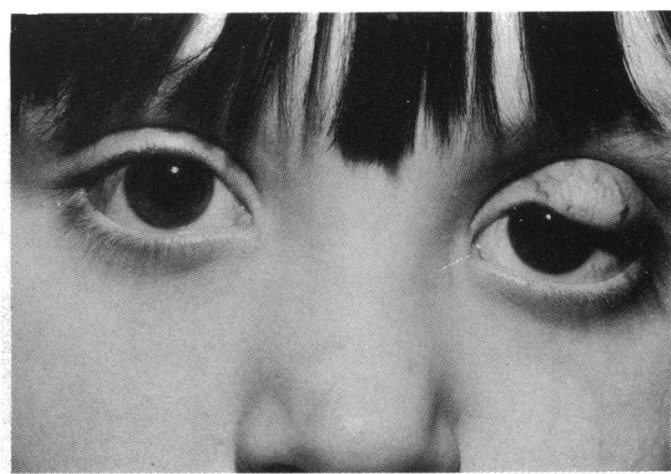

Figure 4A Seven-year-old child with involuted upper lid haemangioma.

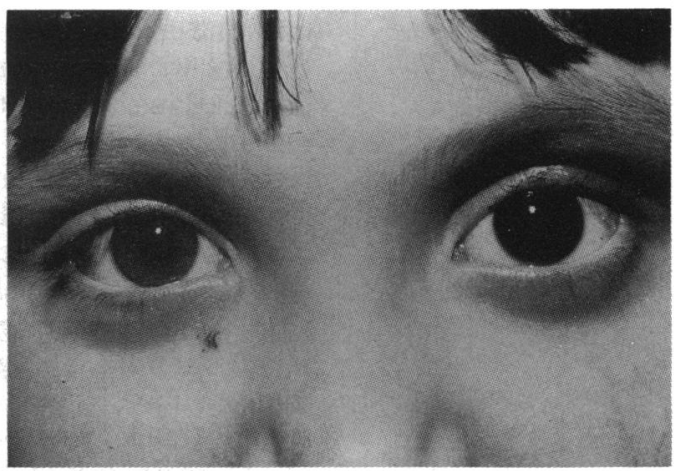

Figure 4B Same child after debulking and full-thickness lid resection of the upper lid haemangioma.

surgical debulking at a later age. This treatment is successful in diminishing tumour size and the risk of amblyopia and is associated with minimal complications.

This paper was presented as part of the Dermot Pierse lecture at the Royal Society of Medicine on 12 September 1990.

1 Haik GR, Jakobiak FA, Ellsworth RM, Jones IS. Capillary Hemangioma of the lids and orbit: an analysis of the clinical features and therapeutic results in 101 cases. Ophthalmology 1979; 86: 760-89.

2 Margileth A, Museles M. Cutaneous Hemangioma in children: diagnosis and conservative management. $\mathcal{F} \mathrm{Am}$ Med Assoc 1965; 194: 523-6.

3 Zarem HA, Edgerton MT, Induced resolution of cavernous Hemangiomas following prednisolone treatment. Plas Reconstr Surg 1967; 39: 76-83.

4 Mazzola RF. Treatment of haemangiomas in children by intralesional injection of steroids. Chirurg Plast (Berlin) 1978 4: 161-71.

5 Kushner BJ. Intralesional cortical steroid injection for infantile adnexal Hemangioma. Am f Ophthalmol 1982; 93: 496-506.

6 Von Noorden GK. Binocular vision in ocular motility. St Louis: Mosby, 1980: 219 .

7 Stigmar G, Crawford JS, Ward, CM, Thompson HG. Oph thalmic sequelae of infantile hemangiomas of the eyelids and orbit. Am f Ophthalmol 1978; 85: 806-13.

8 Kushner BJ. Capillary haemangiomas of the adnexa in infants. Oculoplastic orbital and reconstructive surgery 1: Eyelids. Hornblass A, eds. In: Baltimore: Williams and Wilkins, 1988 243-50.

9 Bonavolonta G, Vassallo P, Uccello G, Tranfa F. Our experience with intralesional cortical steroid injection therapy for infantile adnexal hemangioma. Orbit 1985; 4: 